\title{
Characterizing the chemical space of $\gamma$-secretase inhibitors and modulators
}

Ángel Santiago ${ }^{\mathrm{a}}$, Dulce C. Guzmán-Ocampo ${ }^{\mathrm{a}}$, Rodrigo Aguayo-Ortiz ${ }^{\mathrm{b}, *}$, and Laura Dominguez ${ }^{\mathrm{a}, *}$

${ }^{a}$ Departamento de Fisicoquímica, Facultad de Química, Universidad Nacional Autónoma de México, Mexico City 04510, Mexico

${ }^{b}$ Departamento de Farmacia, Facultad de Química, Universidad Nacional Autónoma de México, Mexico City 04510, Mexico

*Correspondence: Laura Dominguez (lauradd@unam.mx) and Rodrigo Aguayo-Ortiz (odaguayo@,comunidad.unam.mx)

Table of contents

\section{Contents}

Fig. S1 Tanimoto similarity distribution of the 25 molecular fingerprints for the 785 GSIs and GSMs

Fig. S2 Chemical space of GSIs colored by $\mathrm{pIC}_{50}$ potencies

Fig. S3 Chemical space of sulfones colored by $\mathrm{plC}_{50}$ potencies

Fig. S4 Chemical space of NSAID-derived carboxylic acid-containing GSMs colored by $\mathrm{pIC}_{50}$ potencies

Fig. S5 Chemical space of phenylimidazoles colored by $\mathrm{plC}_{50}$ potencies 


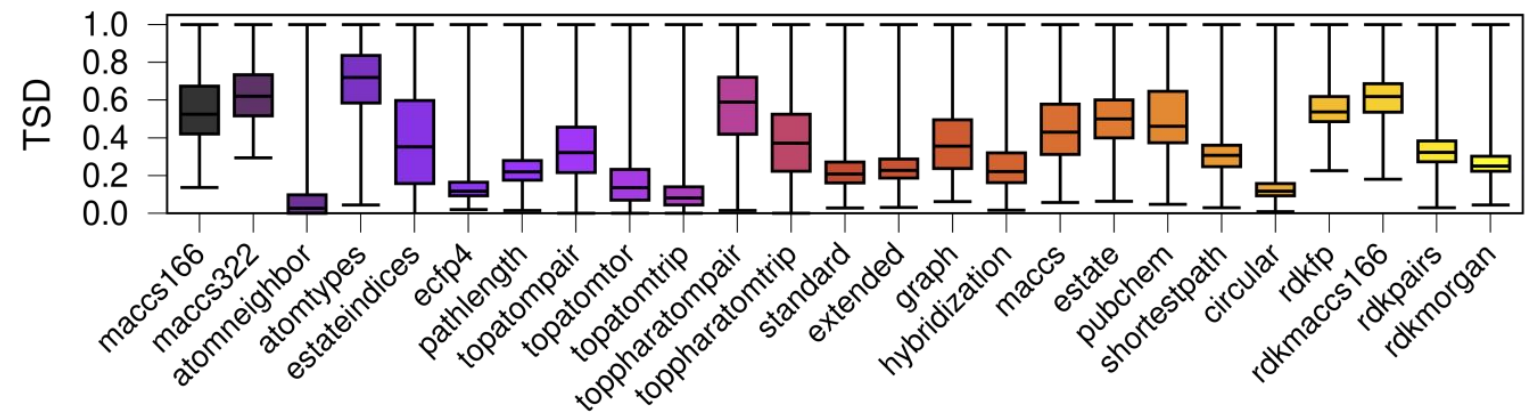

Molecular Fingerprints

Figure S1. Tanimoto similarity distribution (TSD) of the 25 molecular fingerprints for the 785 GSIs and GSMs used in the study.

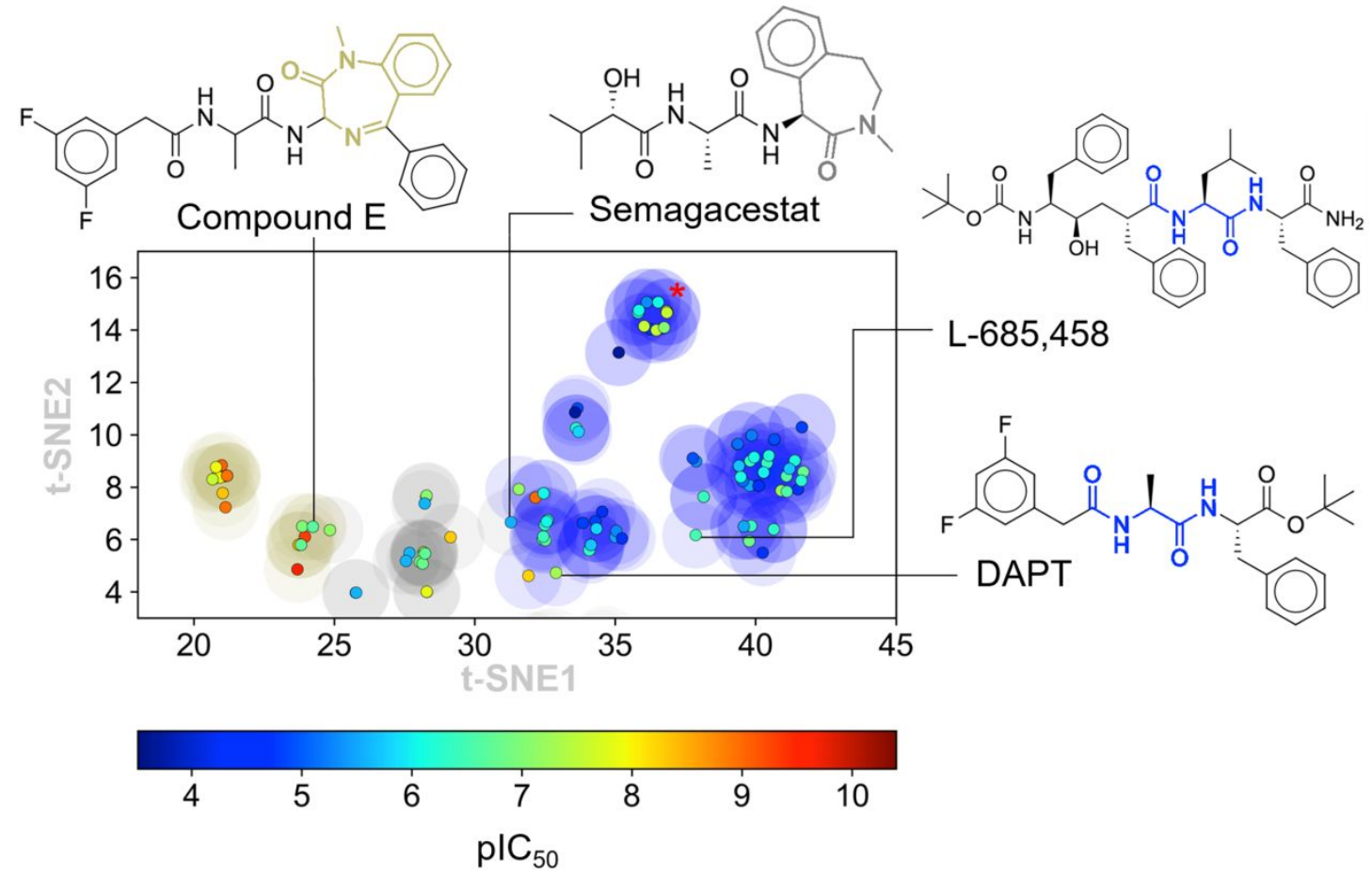

Figure S2. Chemical space of GSIs colored by $\mathrm{pIC}_{50}$ potencies. Same depiction as in Figure 1 using the same color codes in the tSNE projection. The dots are colored in accordance with $\mathrm{A} \beta \mathrm{pIC}_{50}$ values. Red asterisk corresponds to D-helical peptides. Highest is $\mathrm{pIC}_{50}$ value highest is the potency. 


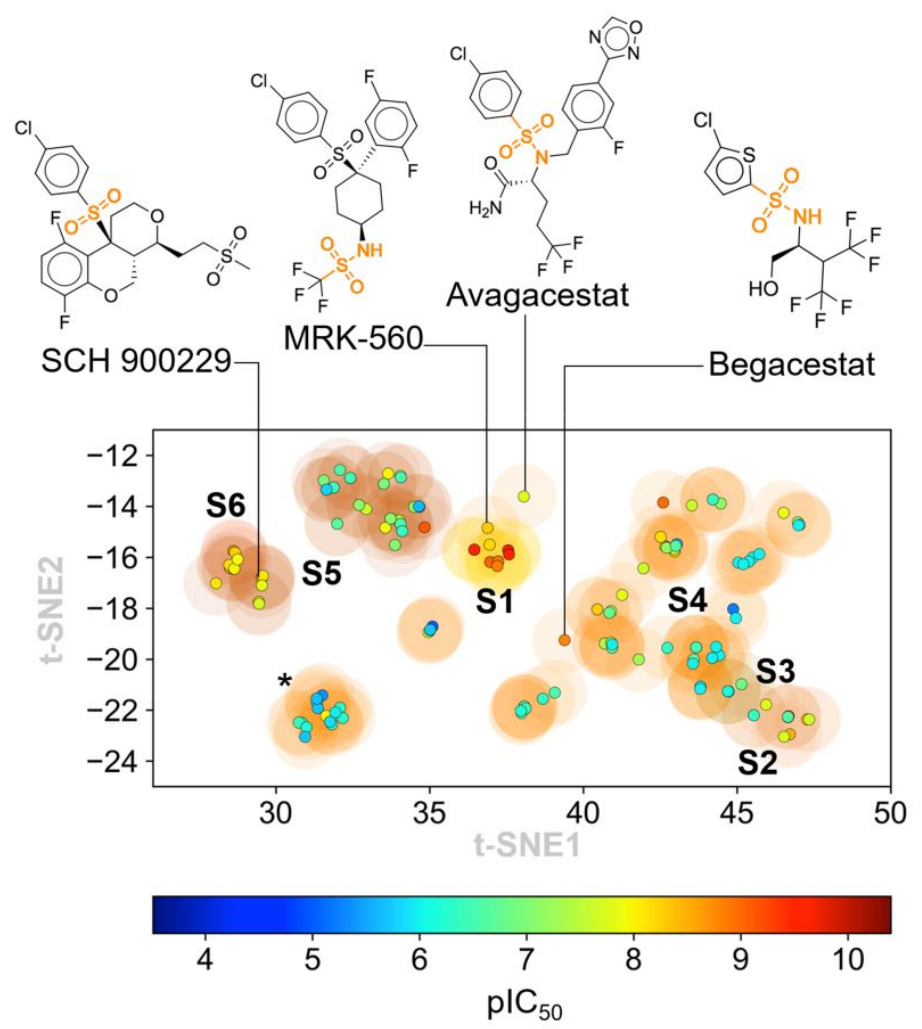

Figure S3. Chemical space of sulfones colored by $\mathrm{pIC}_{50}$ potencies. t-SNE for sulfone classes showing the $\mathrm{A} \beta \mathrm{pIC} \mathrm{C}_{50}$ values. As in Figure 2, the color codes for subclasses as well the structures of representative compounds were used. Although most of $\mathrm{pIC}_{50}$ values for sulfones are reported against $A \beta 40$, some of them are unclear.

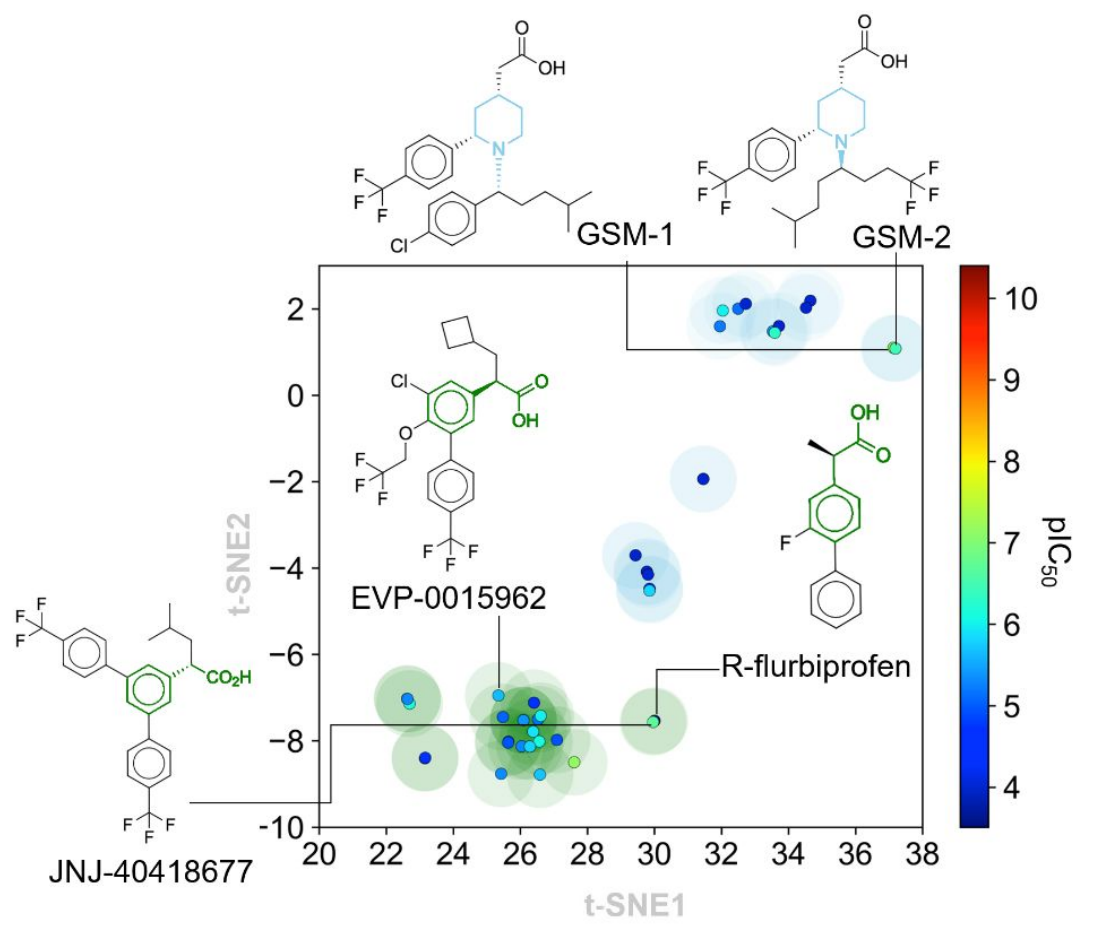

Figure S4. Chemical space of NSAID-derived carboxylic acid-containing GSMs colored by $\mathrm{pIC}_{50}$ potencies. Zoom-in for Flurbiprofen-related and piperidine groups using $\mathrm{A} \beta 42 \mathrm{pIC}_{50}$ values as displayed property. The same color codes for subclasses in $\mathrm{t}$-SNE plot as well the structures of representative compounds were used. 


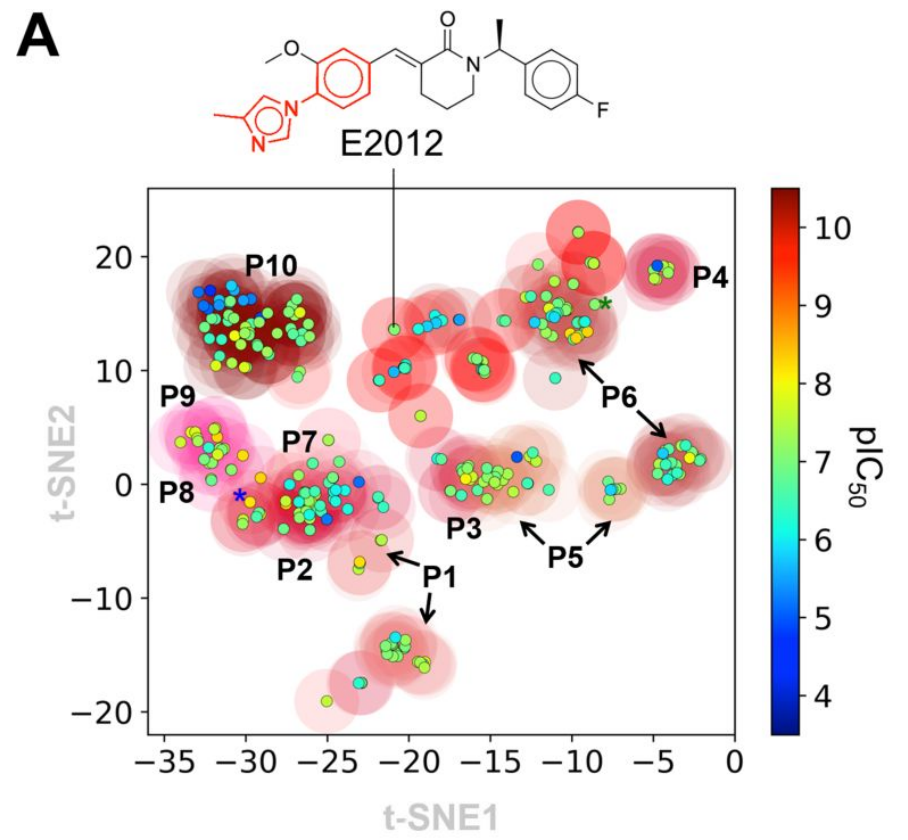

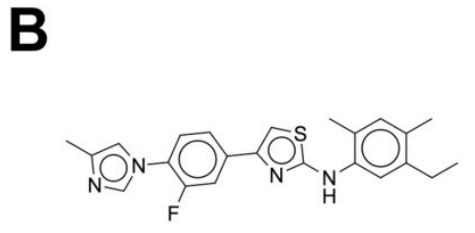

(P1) NGP-555

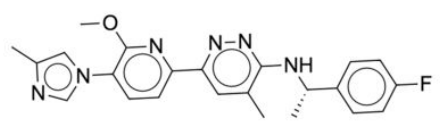

(P2) BPN-15606

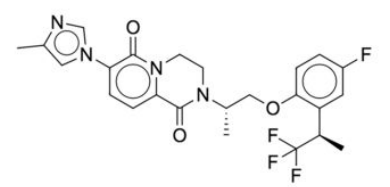

(P6) PF-06442609

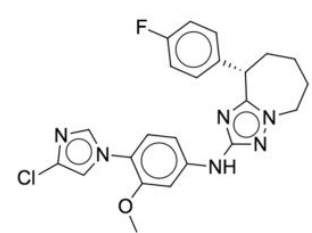

(P2) BMS-869780

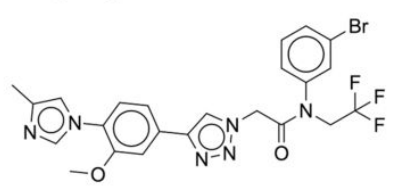

(P3) ST1120

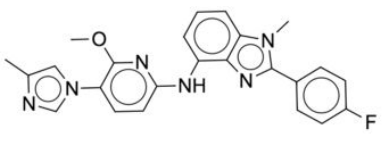

(P8) JNJ-42601572

Figure S5. Chemical space of phenylimidazoles colored by $\mathrm{pIC}_{50}$ potencies. A) Same plot as in Figure 5 for phenylimidazoles subclasses but using $\mathrm{A} \beta 42 \mathrm{pIC}_{50}$ potencies. Blue and green asterisks correspond to BPN-15606 and PF-06648671 compounds, respectively. B) Chemical structures of representative phenylimidazoles: with exception of BPN-15606, the subclass is indicated for each compound.

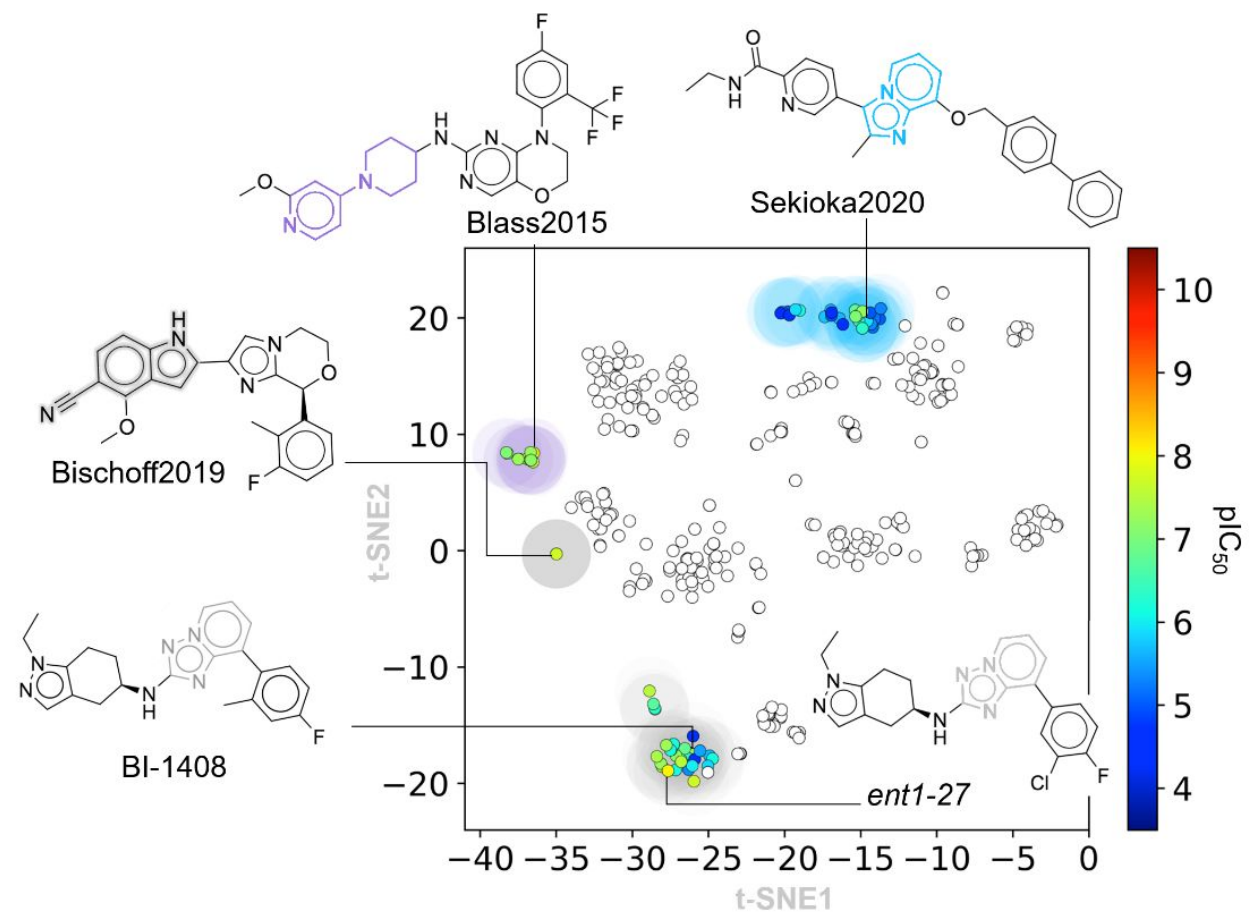

Figure S6. Chemical space of phenylimidazole-related compounds colored by $\mathrm{pIC}_{50}$ potencies. t-SNE plot of phenylimidazolerelated clusters as depicted in Figure 6A. Compounds were colored in accordance with A $\beta 42 \mathrm{pIC}_{50}$ potencies, while color codes for classes were conserved as in Figure 6A. The phenylimidazole groups are displayed as uncolored dots. 